\title{
Backward Bifurcation of Sir Epidemic Model with Non- Monotonic Incidence Rate under Treatment
}

\author{
${ }^{1}$ D. Jasmine and ${ }^{2}$ E. C. Henry Amirtharaj \\ ${ }^{1,2} P G \&$ Research Department of Mathematics Bishop Heber College (Autonomous), Trichy.
}

\begin{abstract}
Treatment is of great importance in fighting against infectious diseases. Backward bifurcation of SIR epidemic model with treatment rate is proposed and analyzed by Wang $W$. We have reinvestigated the above model by considering a backward bifurcation of SIR epidemic model with non-monotone incidence rate under treatment. It is assumed that the treatment rate is proportional to the number of patients as long as this number is below a certain capacity and it becomes constant when that number of patients exceeds this capacity. Mathematical models have become important tools in analyzing the spread and control of infectious diseases. It is shown that this kind of treatment rate leads to the existence of multiple endemic equilibria where the basic reproduction number plays a big role in determining their stability. Moreover, numerical calculations are support to analyze the global stability of unique endemic equilibrium.
\end{abstract}

Keywords: Backward Bifurcation, Basic reproduction number, Endemic, Epidemic, Incidence rate, Treatment function.

\section{Introduction}

The asymptotic behavior of epidemic models has been studied by many researchers. Various epidemic models have been formulated and analyzed by many researchers. In the course of disease control, the treatment is an important method to decrease the spread of diseases. Recently, many researchers have explored the role of treatment function $\mathrm{T}(\mathrm{I})$, which represents the number of infectious individuals of healing, within epidemic disease models. In classical epidemic models, the treatment rate is assumed to be proportional to the number of the infective. Assumed that the capacity for the treatment of a disease in a community is a constant treatment rate $0 \leq \mathrm{r} \leq 1$, in this case the treatment function is rI. i.e., $\mathrm{T}(\mathrm{I})=$ rI. For recent work, using an SIR model, Wang and Ruan [14] supposed that the treatment function has the form

$$
\begin{array}{r}
\mathrm{T}(\mathrm{I})=\mathrm{r}, \text { if } \mathrm{I}>0 \\
0, \text { if } \mathrm{I}=0
\end{array}
$$

Where $r$ is a constant value. They discussed the stability of equilibria and proved the model exhibits Bogdanov-Takens bifurcation, Hopf bifurcation and Homo-clinic bifurcation. Mena Lorca and Hethcote [13] also analyzed an SIRS model with the same saturation incidence. Non-linear incidence rate of the form $\mathrm{bI}^{\mathrm{p}} \mathrm{S}^{\mathrm{q}}$ were investigated by Liu. et. al. [12]. A very general form of non-linear incidence rate was considered by Derrick and Driessche [3]. A more general incidence $\lambda \mathrm{I}^{\mathrm{p}} \mathrm{S} /\left(1+\alpha \mathrm{I}^{\mathrm{q}}\right)$ was proposed by many other researchers $[2,4,6,8,10]$. Xiao and Ruan [15] proposed an epidemic model with non-monotonic incidence rate $\lambda \mathrm{IS} /\left(1+\alpha \mathrm{I}^{2}\right)$. Besides the rate and nature of incidence, treatment plays an important role to control the spread of diseases. This model is investigated and analyzed by Kar and Batabyal [11]. Also this model is modified by Gajendra. et. al. [5]. As the improving of the hospital's treatment conditions, such as effective medicines, skillful techniques, the treatment rate will be increased. At last, the treatment rate of the disease won't increase until a plateau is reached. Therefore, constant recovery is a poor description of real-world infections. Aiming at this kind of improvement of circumstance, Wang incorporated the following piecewise linear treatment function into an SIR model. Wang [1] proposed a treatment function

$$
\mathrm{T}(\mathrm{I})=\underset{\mathrm{r}}{\mathrm{r}}, \text { if } 0 \leq \mathrm{I} \leq \mathrm{I}_{0}
$$

Where $\mathrm{K}_{1}=\mathrm{rI}_{0}$ for some fixed value $\mathrm{I}_{0}$. For classical epidemic models, it is common that a basic reproduction number is a threshold in a sense that a disease is persistent if the basic reproduction number is greater than 1, and dies out if it is below 1 . In this case, the bifurcation leading from a disease free equilibrium to an endemic equilibrium is forward. In recent years, papers found backward bifurcations due to social groups with different susceptibilities, pair formation, macro parasite infection, nonlinear incidences, and age structures in epidemic models. In this case, the basic reproduction number does not describe the necessary elimination effort; rather the effort is described by the value of the critical parameter at the turning point. Thus, it is important to identify backward bifurcations to obtain thresholds for the control of diseases.

The treatment is an important method to decrease the spread of diseases such as measles, tuberculosis and flu. In classical epidemic models, the treatment rate of infective is assumed to be proportional to the number of the infective. This is unsatisfactory because the resources for treatment should be quite large. In 
fact, every community should have a suitable capacity for treatment. If it is too large, the community pays for unnecessary cost. If it is too small, the community has the risk of the outbreak of a disease. Thus, it is important to determine a suitable capacity for the treatment of a disease, we adopt a constant treatment, which simulates a limited capacity for treatment. Note that a constant treatment is suitable when the number of infective is large. In this paper, we modify it into

$$
T(I)= \begin{cases}\mathrm{r}, & \text { if } 0 \leq \mathrm{I} \leq I_{0}, \\ \mathrm{k}, & \text { if } \mathrm{I}>\mathrm{I}_{0},\end{cases}
$$

where, $\mathrm{k}=\mathrm{rI}_{0}$. This means that the treatment rate is proportional to the number of the infective when the capacity of treatment is not reached, and otherwise, takes the maximal capacity. This renders for example the situation where patients have to be hospitalized: the number of hospital beds is limited. This is true also for the case where medicines are not sufficient. Evidently, this improves the classical proportional treatment and the constant treatment.

We will consider a population that is divided into three types: susceptible, infective and recovered. Let $\mathrm{S}$, I and R denote the numbers of susceptive, infective, recovered individuals, respectively.

\section{Mathematical Model}

Then the model to be studied takes the following form:

$$
\begin{aligned}
& \frac{d s}{d t}=a-d S-\frac{\lambda S I}{1+\alpha I}, \\
& \frac{d I}{d t}=\frac{\lambda S I}{1+\alpha I}-(d+m+\varepsilon) I-T(I) \\
& \frac{d R}{d t}=m I-d R+T(I),
\end{aligned}
$$

where $\mathrm{a}$ is the recruitment rate of the population, $\mathrm{d}$ the natural death rate of the population, $\lambda$ is the proportionality constant, $m$ is the natural recovery rate of the infective individuals, $\alpha$ is the parameter measures of the psychological or inhibitory effect. It is assumed that all the parameters are positive constants. Clearly, $R_{3}^{+}$is positively invariant for system (2). Since the first two equations in (2) are independent of the variable $\mathrm{R}$, it suffices to consider the following reduced model:

$$
\begin{aligned}
& \frac{d S}{d t}=a-d S-\frac{\lambda S I}{1+\alpha I}, \\
& \frac{d I}{d t}=\frac{\lambda S I}{1+\alpha I}-(d+m+\varepsilon) I-T(I),
\end{aligned}
$$

The purpose of this paper is to show that (3) has a backward bifurcation if the capacity for treatment is small. We will prove that (3) has bi-stable endemic equilibrium if the capacity is low. The organization of this paper is as follows. In the next section, we study the bifurcations of (3). In Section 5 we present a global analysis and simulations of the model.

\section{Equilibrium}

In this section, we first consider the equilibria of (3) and their local stability. $E_{0}=(A / d, 0)$ is a disease free equilibrium. An endemic equilibrium of (3) satisfies

$$
\begin{aligned}
& a-d S-\frac{\lambda S I}{1+\alpha I}=0 \\
& \frac{\lambda S I}{1+\alpha I}-(d+m+\varepsilon) I-T(I)=0
\end{aligned}
$$

When $0<I \leq I_{0}$, system (4) becomes 


$$
\begin{aligned}
& a-d S-\frac{\lambda S I}{1+\alpha I}=0 \\
& \frac{\lambda S I}{1+\alpha I}-(d+m+\varepsilon+r) I=0
\end{aligned}
$$

(5)

When $\mathrm{I}>\mathrm{I}_{0}$, the system (4) becomes

$$
\begin{aligned}
& a-d S-\frac{\lambda S I}{1+\alpha I}=0 \\
& \frac{\lambda S I}{1+\alpha I}-(d+m+\varepsilon) I-k=0
\end{aligned}
$$

$$
\text { Let } \quad \mathrm{R}_{0}=\frac{\lambda a}{d(d+m+\varepsilon+r) \text {. }}
$$

Then $\mathrm{R}_{0}$ is a basic reproduction number of (3). If $\mathrm{R}_{0}>1$, (5) admits a unique positive solution of $\mathrm{E}^{*}=\left(\mathrm{S}^{*}, \mathrm{I}^{*}\right)$ where

$$
\begin{gathered}
\frac{\lambda S^{*} I^{*}}{1+\alpha I^{*}}-(d+m+\varepsilon+r) I^{*}=0 \\
\lambda S^{*}=(d+m+\varepsilon+r)\left(1+\alpha I^{*}\right) \\
S^{*}=\frac{(d+m+\varepsilon+r)\left(1+\alpha I^{*}\right)}{\lambda}, \\
a-d S^{*}-\frac{\lambda S^{*} I^{*}}{1+\alpha I^{*}}=0 \\
a-\frac{d(d+m+\varepsilon+r)\left(1+\alpha I^{*}\right)}{\lambda}-\lambda \frac{(d+m+\varepsilon+r)\left(1+\alpha I^{*}\right)}{\lambda\left(1+\alpha I^{*}\right)} I^{*}=0 \\
\Rightarrow I^{*}=\frac{\lambda a-d(d+m+\varepsilon+r)}{(\alpha d+\lambda)(d+m+\varepsilon+r)}
\end{gathered}
$$

Using the definition of $R_{0}$, we get

$$
\mathrm{I}^{*}=\frac{d\left(R_{0}-1\right)}{\alpha d+\lambda}
$$

Clearly, $\mathrm{E}^{*}$ is an endemic equilibrium of (3) if and only if

$$
\begin{gathered}
1<I_{0} \leq \frac{d\left(R_{0}-1\right)}{\alpha d+\lambda} \\
\text { i.e., } 1<I_{0} \leq \frac{\left(R_{0}-1\right)}{\alpha+\frac{\lambda}{d}} \\
1<I_{0} \leq \frac{R_{0}}{\alpha+\frac{\lambda}{d}}-\frac{1}{\alpha+\frac{\lambda}{d}} \\
1<I_{0}+\frac{1}{\alpha+\frac{\lambda}{d}} \leq \frac{R_{0}}{\alpha+\frac{\lambda}{d}} \\
1<R_{0} \leq \frac{\alpha d+\lambda}{d}\left(I_{0}+\frac{d}{\alpha d+\lambda}\right)
\end{gathered}
$$




$$
1<R_{0} \leq 1+\frac{\alpha d+\lambda}{d} I_{0}
$$

In order to obtain positive solution of system (6), we solve $\mathrm{S}$ from the first equation of (6) to obtain $\mathrm{S}$,

$$
\begin{aligned}
& a-d S-\frac{\lambda S I}{1+\alpha I}=0 \\
& a-S\left(d+\frac{\lambda I}{1+\alpha I}\right)=0 \\
& a-S \frac{d(1+\alpha I)+\lambda I}{1+\alpha I} \\
& S=\frac{a(1+\alpha I)}{d(1+\alpha I)+\lambda I}
\end{aligned}
$$

When $\mathrm{I}>\mathrm{I}_{0}$, system (4) becomes

$$
\begin{aligned}
& \frac{\lambda S I}{1+\alpha I}-(d+m+\varepsilon) I-k=0 \\
& \lambda\left(\frac{a(1+\alpha I)}{d(1+\alpha I)+\lambda I}\right) I-(d+m+\varepsilon) I-k=0
\end{aligned}
$$

$[(\alpha d+\lambda)(d+m+\varepsilon)-\lambda a \alpha] I^{2}-\left[d^{2}+d m+d \varepsilon+k(\alpha d+\lambda)-\lambda a\right] I+k d=0$

where

$$
\text { i.e., } \quad A I^{2}+B I+C=0
$$

$$
B=d^{2}+d m+d \varepsilon+k(\alpha d+\lambda)-\lambda a .
$$

If $\mathrm{B} \geq 0$, it clear that (8) does not have a positive solution. Let us consider the case where $\mathrm{B}<0$.

If $\Delta=B^{2}-4[(\alpha d+\lambda)(d+m+\varepsilon)-\lambda a \alpha] d k$, it is easy to obtain

$$
\Delta=\left[d^{2}+d m+d \varepsilon+k(\alpha d+\lambda)-\lambda a\right]^{2}-4[(\alpha d+\lambda)(d+m+\varepsilon)-\lambda a \alpha] d k
$$

$\Delta=\left[-R_{0} d(d+m+\varepsilon+r)+d^{2}+d m+d \varepsilon+k(\alpha d+\lambda)\right]^{2}-4[(\alpha d+\lambda)(d+m+\varepsilon)-\lambda a \alpha] d k$ where

$$
\lambda a=R_{0} d(d+m+\varepsilon+r)
$$

It follows that $\Delta \geq 0$ is equivalent to

$$
R_{0} \geq 1+\frac{k(\alpha d+\lambda)-d r}{d(d+m+\varepsilon+r)}+2 \frac{\sqrt{(\alpha d+\lambda)(d+m+\varepsilon) k d}}{d(d+m+\varepsilon+r)} \cong p_{0}
$$

(or)

$$
R_{0} \leq 1+\frac{k(\alpha d+\lambda)-d r}{d(d+m+\varepsilon+r)}-2 \frac{\sqrt{(\alpha d+\lambda)(d+m+\varepsilon) k d}}{d(d+m+\varepsilon+r)} .
$$

Note that $\mathrm{B}<0$ is equivalent to

$$
R_{0}>1+\frac{k(\alpha d+\lambda)-d r}{d(d+m+\varepsilon+r)} .
$$

It follows that $\mathrm{B}<0$ and $\Delta \geq 0$ if and only if (9) holds. Equation (8) implies

$$
\begin{aligned}
& {[(\alpha d+\lambda)(d+m+\varepsilon)-\lambda a \alpha] I^{2}+B I+d k=0} \\
& I=\frac{-B \pm \sqrt{B^{2}-4(\alpha d+\lambda)(d+m+\varepsilon) d k}}{2[(\alpha d+\lambda)(d+m+\varepsilon)-\lambda a \alpha]} \\
& I=\frac{-B \pm \sqrt{\Delta}}{2[(\alpha d+\lambda)(d+m+\varepsilon)-\lambda a \alpha]}
\end{aligned}
$$

Then (8) has two positive solutions $\mathrm{I}_{1}$ and $\mathrm{I}_{2}$ where 


$$
\begin{aligned}
& I_{1}=\frac{(-B-\sqrt{\Delta})}{2[(\alpha d+\lambda)(d+m+\varepsilon)-\lambda a \alpha]} \\
& I_{2}=\frac{-B+\sqrt{\Delta}}{2[(\alpha d+\lambda)(d+m+\varepsilon)-\lambda a \alpha]}
\end{aligned}
$$

Set $S_{i}=\frac{a(1+\alpha I)}{d(1+\alpha I)+\lambda I}$ and $\mathrm{E}_{\mathrm{i}}=\left(\mathrm{S}_{\mathrm{i}}, \mathrm{I}_{\mathrm{i}}\right)$ for $\mathrm{i}=1,2$. Then $\mathrm{E}_{\mathrm{i}}$ is an endemic equilibrium of (3) if $I_{i} \geq I_{0}$. Let us consider the condition under which $\mathrm{I}_{1}>\mathrm{I}_{0}$. By the definitions, we see that

$$
\begin{gathered}
\mathrm{I}_{0}<\mathrm{I}_{1}=\frac{(-B-\sqrt{\Delta})}{2[(\alpha d+\lambda)(d+m+\varepsilon)-\lambda a \alpha]} \\
\mathrm{I}_{0}<\frac{(-B-\sqrt{\Delta})}{2[(\alpha d+\lambda)(d+m+\varepsilon)-\lambda a \alpha]} \\
2[(\alpha d+\lambda)(d+m+\varepsilon)-\lambda a \alpha] I_{0}<-B-\sqrt{\Delta} \\
-B-\sqrt{\Delta}>2[(\alpha d+\lambda)(d+m+\varepsilon)-\lambda a \alpha] I_{0} .
\end{gathered}
$$

This implies that

$$
\begin{aligned}
& -\sqrt{\Delta}>B+2[(\alpha d+\lambda)(d+m+\varepsilon)-\lambda a \alpha] I_{0} . \\
& B+2[(\alpha s+\lambda)(d+m+\varepsilon)-\lambda a \alpha] I_{0}<0 .
\end{aligned}
$$

It follows from the definition of $\mathrm{B}$ that

$$
R_{0}>1+\frac{k(\alpha d+\lambda)-d r}{d(d+m+\varepsilon+r)}+2 \frac{(\alpha d+\lambda)(d+m+\varepsilon) I_{0}}{d(d+m+\varepsilon+r)} \cong p_{1} .
$$

Further, (13) implies that

$$
\left[B+2(\alpha d+\lambda)(d+m+\varepsilon) I_{0}\right]^{2}>\Delta .
$$

By direct calculation, we see that (16) is equivalent to

$$
R_{0}<1+\frac{(\alpha d+\lambda) I_{0}}{d} \cong p_{2} .
$$

Hence $\mathrm{I}_{1}>\mathrm{I}_{0}$ holds if and only if (15) and (17) are valid. Moreover, if $\mathrm{R}_{0} \leq p_{1}$ or $\mathrm{R}_{0} \geq p_{2}$.

We have $\mathrm{I}_{1} \leq \mathrm{I}_{0}$. By similar arguments as above, we see that $\mathrm{I}_{0}<\mathrm{I}_{2}$ if (15) holds, or

$$
p_{2}<R_{0} \leq P_{1} \text {. }
$$

Furthermore,

$$
\begin{aligned}
& I_{2} \leq I_{0} \text { if } \\
& R_{0} \leq \min \left\{p_{1}, p_{2}\right\} .
\end{aligned}
$$

Summarizing the discussion above, we have the following conclusions.

\section{Theorem: 4.1}

\section{Some Theorems}

$E^{*}=\left(\mathrm{S}^{*}, \mathrm{I}^{*}\right)$ is an endemic equilibrium of (3) if and only if $1<R_{0} \leq p_{2}$. Furthermore, $\mathrm{E}^{*}$ is the unique endemic equilibrium of (3) if $1<R_{0} \leq P_{2}$ and one of the following conditions are satisfied

(i) $\quad R_{0}<p_{0}$

(ii) $\quad p_{0} \leq R_{0}<p_{1}$.

Note that $d r>(\alpha d+\lambda)(d+m+\varepsilon) I_{0}$ is equivalent to that $p_{1}<p_{2}$.

Theorem: 4.2

Endemic equilibrium $\mathrm{E}_{1}$ and $\mathrm{E}_{2}$ do not exist if $R_{0}<p_{0}$. Further, if $R_{0} \geq p_{0}$, we have the following: 
i) If $d r>(\alpha d+\lambda)(d+m+\varepsilon) I_{0}$, then both $E_{1}=\left(S_{1}, I_{1}\right)$ and $E_{2}=\left(S_{2}, I_{2}\right)$ exist when $p_{1}<R_{0}<p_{2}$.

ii) If $d r>(\alpha d+\lambda)(d+m+\varepsilon) I_{0}$, then $\mathrm{E}_{1}$ does not exist but $\mathrm{E}_{2}$ exist if when $R_{0} \geq p_{2}$.

iii) Let $d r \leq(\alpha d+\lambda)(d+m+\varepsilon) I_{0}$, Then $\mathrm{E}_{1}$ does not exist. Further, $\mathrm{E}_{2}$ exist when $p_{2}<R_{0}$ and $\mathrm{E}_{2}$ does not exist when $R_{0} \leq p_{2}$.

We consider $p_{0}>1$. If $d r \geq(\alpha d+\lambda)(d+m+\varepsilon) I_{0}$, a typical bifurcation diagram is illustrated in figure 1 , where the bifurcation from the disease free equilibrium at $\mathrm{R}_{0}=1$ is forward and there is a backward bifurcation from an endemic equilibrium at $\mathrm{R}_{0}=1.5$, which gives rise to the existence of multiple endemic equilibrium.

Further, if $d r<(\alpha d+\lambda)(d+m+\varepsilon) I_{0}$, a typical bifurcation diagram is illustrated in Fig. 2, where the bifurcation at $R_{0}=1$ is forward and (3) has one unique endemic equilibrium for all $R_{0}>1$.

Note that a backward bifurcation with endemic equilibrium when $R_{0}<1$ is very interesting in applications. We present the following corollary to give conditions for such a backward bifurcation to occur.

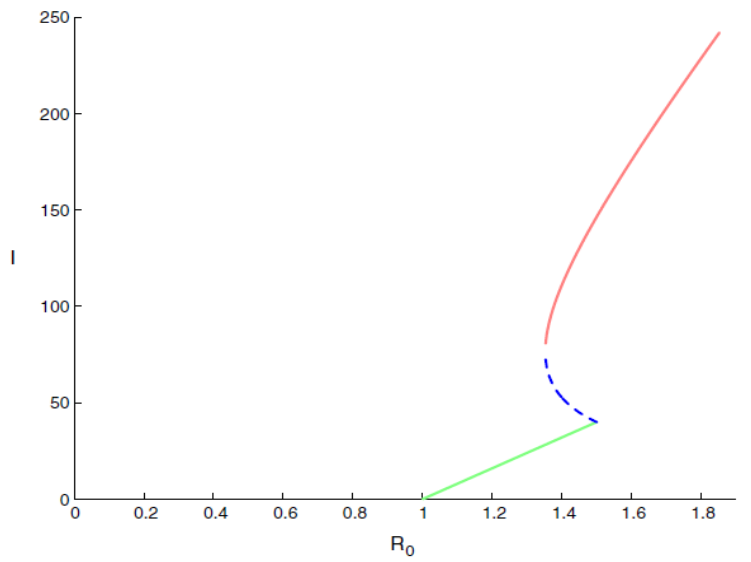

Fig. 1

The figure of infective sizes at equilibrium versus $\mathrm{R}_{0}$ when $\mathrm{I}_{0}=40, \mathrm{~m}=0.01, \varepsilon=0.01$, $\mathrm{d}=0.8, \mathrm{r}=1.5$, where (i) of theorem 4.2 holds. The bifurcation from the disease free equilibrium at $\mathrm{R}_{0}=1$ is forward and there is a backward bifurcation from an endemic equilibrium at $\mathrm{R}_{0}=1.5$, which leads to the existence of multiple endemic equilibrium.

Corollary: 4.3

System (3) has a backward bifurcation with endemic equilibrium when $\mathrm{R}_{0}<1$ if $d r>(\alpha d+\lambda)(d+m+\varepsilon) I_{0}$, and $\mathrm{p}_{0}<1$.

Proof: Example:

This corollary is a simple consequence of (i) of theorem 4.2

Fix $\mathrm{I}_{0}=20, \lambda=0.01, \varepsilon=0.01, \mathrm{~d}=1, \alpha=0.1, \mathrm{k}=0.2, \mathrm{~m}=0.3$ and $\mathrm{r}=6 . \quad$ Then $p_{0} \approx 0.2060, p_{1} \approx 0.9707, p_{2}=3.2$ and $\quad d r-(\alpha d+\lambda)(d+m+\varepsilon) I_{0}=3.12$. Thus, (3) has a backward bifurcation with endemic equilibrium this case (by fig3).

As $\mathrm{I}_{0}$ (the capacity of treatment resources) increases, by the definition we see that $\mathrm{p}_{0}$ increases. When $\mathrm{I}_{0}$ is so large that $\mathrm{p}_{0}>1$, it follows from Theorem 3.2 that there is no backward bifurcation with endemic equilibrium when $\mathrm{R}_{0}<1$. If we increase $\mathrm{I}_{0}$ to $R_{0}<p_{0}$, (3) does not have a backward bifurcation because endemic equilibrium $E_{1}$ and $E_{2}$ do not exist. This means that an insufficient capacity for treatment is a source of the backward bifurcation. 


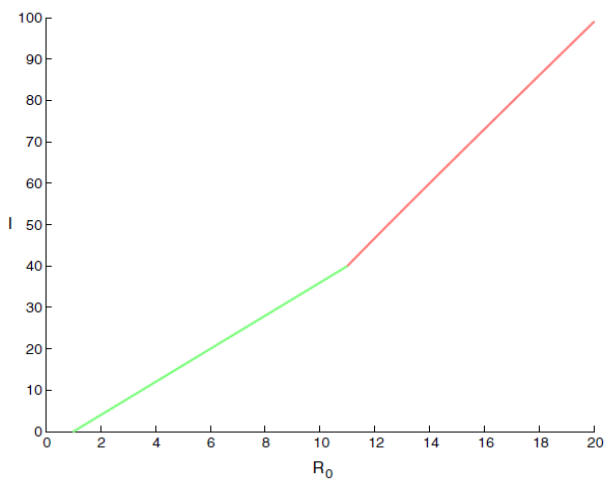

Fig. 2

The diagram of $\mathrm{I}^{*}, \mathrm{I}_{2}$ versus $\mathrm{R}_{0}$ when $\mathrm{I}_{0}=40, \lambda=0.2, m=0.1, \varepsilon=0.01, \mathrm{~d}=0.8, \mathrm{r}=0.6$, where (iii) of Theorem 4.2 holds. The bifurcation at $R_{0}=1$ is forward and (3) has a unique endemic equilibrium for $R_{0}>1$.

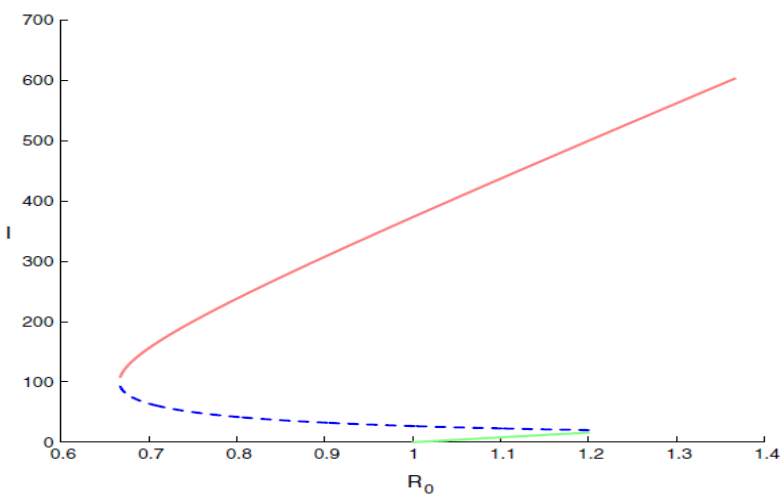

Fig. 3

The figure of $I^{*}, I_{1}$ and $I_{2}$ versus $R_{0}$ that shows a backward bifurcation with endemic equilibrium when $\mathrm{R}_{0}<1$, where Corollary 4.3 holds.

Theorem: 4.4

$E_{0}$ is asymptotically stable if $R_{0}<1$ and $R_{0}>1 . E^{*}$ is asymptotically stable if $I^{*}<I_{0} . E_{1}$ is saddle whenever it exists. For $E_{2}$, we have

i) $E_{2}$ is stable if either

$$
\begin{aligned}
& (\alpha d+\lambda) a-3 d^{2}-d(m+\varepsilon)-2 d^{3} /(m+\varepsilon) \leq(\alpha d+\lambda) k \\
& \left\{\begin{array}{l}
(\alpha d+\lambda) k<(\alpha d+\lambda) a-3 d^{2}-d(m+\varepsilon)-2 d^{3} /(m+\varepsilon) \\
(\alpha d+\lambda) k<\frac{1}{2}\left[2(\alpha d+\lambda) a+(2 d+m+\varepsilon)(d+m+\varepsilon)\left(1-\sqrt{1+\frac{4(\alpha d+\lambda) a}{(d+m+\varepsilon)^{2}}}\right)\right]
\end{array}\right.
\end{aligned}
$$

(21)

ii) $E_{2}$ is unstable if

$$
\left\{\begin{array}{l}
(\alpha d+\lambda) k<(\alpha d+\lambda) a-3 d^{2}-d(m+\varepsilon)-2 d^{3} /(m+\varepsilon), \\
(\alpha d+\lambda) k<\frac{1}{2}\left[2(\alpha d+\lambda) a+(2 d+m+\varepsilon)(d+m+\varepsilon)\left(1-\sqrt{1+\frac{4(\alpha d+\lambda) a}{(d+m+\varepsilon)^{2}}}\right)\right]
\end{array}\right.
$$




\section{Global Analysis and Simulation}

Theorem: 5.1

We begin from the global stability of the disease free equilibrium $\mathrm{E}_{0}$

The disease free equilibrium $\mathrm{E}_{0}$ is globally stable, i.e., the disease dies out, if one of the following conditions is satisfied:

(ii) $\quad R_{0}<1, p_{0}<1$ and $p_{1} \geq 1$

Proof

$R_{0}<1$ implies that $E^{*}$ does not exist. Suppose $p_{0} \geq 1$. It follows from the Theorem 4.2 that $\mathrm{E}_{1}$ exists or $\mathrm{E}_{2}$ exist only if $\mathrm{R}_{0}>\mathrm{p}_{0}$, which is possible since we have $R_{0}<1$. Let us now suppose $\mathrm{p}_{0}<1$ and $p_{1} \geq 1$. If $d r>(\alpha d+\lambda)(d+m+\varepsilon) I_{0}$, since $p_{1}<p_{2}$, it follows from the discussions for (i), (ii) of Theorem 4.2 that $E_{1}$ or $E_{2}$ exists only if $R_{0}>p_{1}$, which is impossible since we have $R_{0}<1$. If $d r \leq(\alpha d+\lambda)(d+m+\varepsilon) I_{0}$, since $1<p_{2}$, it follows from (iii) of Theorem 4.2 that $E_{1}$ and $E_{2}$ do not exist. In summary, endemic equilibrium do not exist under the assumptions.

It is easy to verify that positive solutions of (3) are ultimately bounded. Note that the nonnegative Saxis is positively invariant and that the non negative I-axis repels positive solutions of (3). Since $\mathrm{E}_{0}$ is asymptotically stable, it follows from the Bendixson Theorem that every positive solution of (3) approaches $\mathrm{E}_{0}$ as $t$ approaches infinity. The limit cycles of (3) play crucial roles on the structure of dynamical behaviors of the model. For example, if there is no limit cycle and its endemic equilibrium is unique, the unique endemic equilibrium is globally stable. For this reason, we adopt Dulac functions to obtain conditions for the nonexistence of a limit cycle in (3). We denote the right-hand sides of (3) by $f_{1}$ and $f_{2}$. Then we get

then (3) does not have a limit cycle.

$$
\frac{\partial\left(D f_{1}\right)}{\partial S}+\frac{\partial\left(D f_{2}\right)}{\partial I}<0
$$

\section{Theorem: 5.2}

Proof

System (3) does not have a limit cycle if $r<d$. the region

By the first equation of (3), it is easy to see that positive solutions of (3) eventually enter and remain in

$$
P:=\left\{(S, I): S \leq \frac{a}{d}\right\}
$$

Thus, a limit cycle, if it exists, must lie in the region P. Take a Dulac function $D=\frac{1}{S I}$.

Then we have

$$
\frac{\partial\left(D f_{1}\right)}{\partial S}+\frac{\partial\left(D f_{2}\right)}{\partial I}=-\frac{a}{S^{2} I}<0
$$

if $0<I<I_{0}$. If $I>I_{0}$, it is easy to see that

$$
\frac{\partial\left(D f_{1}\right)}{\partial S}+\frac{\partial\left(D f_{2}\right)}{\partial I}=-\frac{a}{S^{2} I}+\frac{k}{S I^{2}}=\frac{1}{S^{2} I^{2}}(-a I+k S) \leq \frac{a I_{0}}{d S^{2} I^{2}}(r-d)<0
$$

Hence (3) does not have a limit cycle.

Theorem 5.2 implies that there is no limit cycle in (3) if the treatment rate is less than the death rate. We now present a different condition for the nonexistence of limit cycles of (3) which is independent of the treatment

\section{Theorem: $\mathbf{5 . 3}$}

System (3) does not have a limit cycle if

$$
(\alpha d+\lambda) a<(2 d+m+\varepsilon) d .
$$

Proof

We consider a Dulac function D defined by, 


$$
D= \begin{cases}\frac{1}{\mathrm{SI}}, & \text { if } \quad I \leq I_{0}, \\ \frac{1}{S I_{0}}, & \text { if } \quad I>I_{0},\end{cases}
$$

Then we have

$$
\frac{\partial\left(D f_{1}\right)}{\partial S}+\frac{\partial\left(D f_{2}\right)}{\partial I}=-\frac{a}{S^{2} I}<0
$$

if $0<I<I_{0}$. Note that (23) implies that

$$
\begin{aligned}
{\left[-a+(\alpha d+\lambda) S^{2}-(d+m+\varepsilon) S\right]_{S=a / d} } & =-a+(\alpha d+\lambda)\left(\frac{a}{d}\right)^{2}-(d+m+\varepsilon)\left(\frac{a}{d}\right) \\
& =-a+\alpha a^{2}+\frac{\lambda a^{2}}{d^{2}}-a-\frac{m a}{d}-\frac{\varepsilon a}{d} \\
& =\frac{-a d^{2}+\alpha a^{2} d^{2}+\lambda a^{2}-a d^{2}-m a d-\varepsilon a d}{d^{2}} \\
& =\frac{-a\left(d^{2}-\alpha a d^{2}-\lambda a+d^{2}+m d+\varepsilon d\right)}{d^{2}} \\
{\left[-a+(\alpha d+\lambda) S^{2}-(d+m+\varepsilon) S\right]_{S=a / d} } & =\frac{-a\left(d^{2}-\alpha a d^{2}-\lambda a+d^{2}+m d+\varepsilon d\right)}{d^{2}}<0
\end{aligned}
$$

It follows that for $I>I_{0}$

$$
\frac{\partial\left(D f_{1}\right)}{\partial S}+\frac{\partial\left(D f_{2}\right)}{\partial I}=\frac{1}{S^{2} I_{0}}\left[-a+(\alpha d+\lambda) S^{2}-(d+m+\varepsilon) S\right]<0
$$

where the domain P is considered. Therefore, (3) does not have a limit cycle.

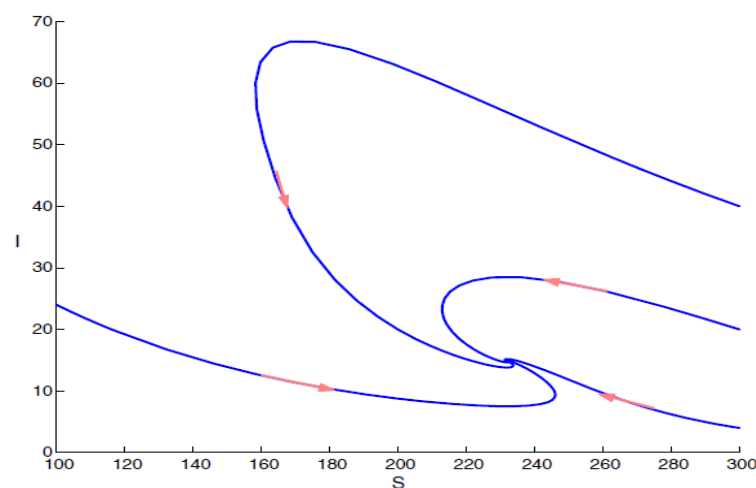

Fig. 4

$\mathrm{E}^{*}$ is globally stable when $a=220, \mathrm{I}_{0}=40, \lambda=0.01, \mathrm{~m}=0.01, \varepsilon=0.01$, $\mathrm{d}=0.8, \mathrm{k}=0.01, \mathrm{r}=1.5$ and $\alpha=0.2$ 


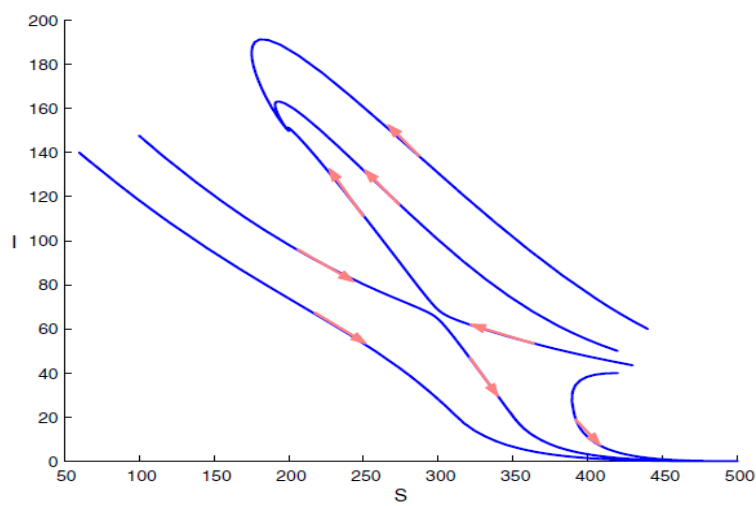

Fig. 5

One region of disease persistence and one region of disease extinction where $a=500, \mathrm{I}_{0}=20, \mathrm{k}=0.01, \varepsilon=0.1, \lambda=0.01 m=0.1, \mathrm{~d}=1$ and $\mathrm{r}=6$, where Corollary 2.3 holds.

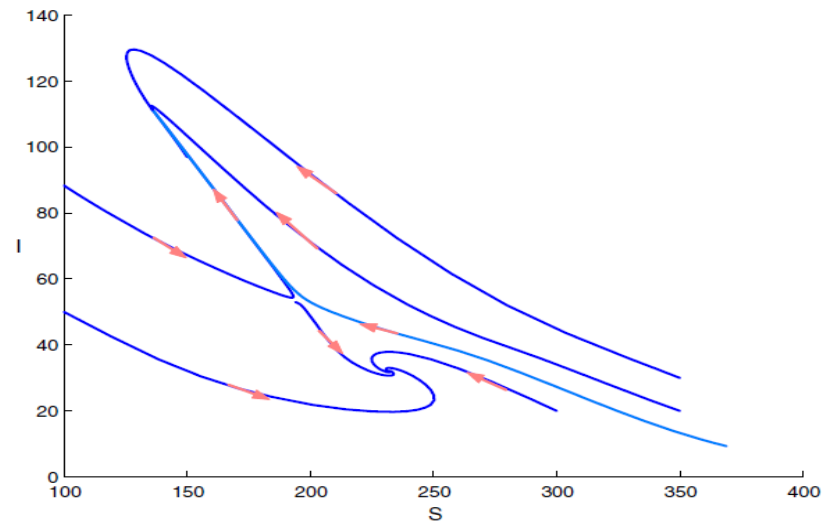

Fig.6

A bi-stable case where endemic equilibria $\mathrm{E}^{*}$ and $\mathrm{E}_{2}$ are stable when $a=260, \mathrm{I}_{0}=40$, $\lambda=0.01, m=0.01, \varepsilon=0.01, \mathrm{~d}=0.8, \mathrm{r}=1.5$, where (i) of theorem 4.2 holds.

If the conditions of theorem 4.1, 5.2 or 5.3 are satisfied, then $E^{*}$ is one unique endemic equilibrium and is globally stable. Numerical calculations shows that the unique endemic equilibrium $\mathrm{E}^{*}$ is also globally stable if Theorems 5.3 and 5.4 are not satisfied (see Fig. 5). If $\mathrm{R}_{0}>\mathrm{p}_{2}$. Theorem 4.2 implies that $\mathrm{E}_{2}$ is a unique endemic equilibrium of (3). Numerical calculations shows that this unique endemic equilibrium is also globally stable. However, by direct calculations, we see that $I_{2}>I^{*}$ if $R_{0}>p_{2}$. This means that we have more infected members in the limited resources for treatment than those with the unlimited resources for treatment. Let the conditions of Corollary 4.3 hold. If $\mathrm{R}_{0}<1$, the stable manifolds of the saddle $\mathrm{E}_{1}$ split $\mathrm{R}_{0}$ into two regions. The disease is persistent in the upper region and dies out in the lower region (see Fig. 6).

The stable manifolds of the saddle $\mathrm{E}$ split the feasible region into two parts; positive orbits in the lower part approach to the endemic equilibrium $\mathrm{E}^{*}$ and positive orbits in the upper part approach to the endemic equilibrium $E^{*}$. Thus, we have a bi-stable case.

\section{Concluding Remarks}

In this paper, we have proposed an epidemic model to simulate the limited resources for the treatment of patients, which can occur because patients have to be hospitalized but there are limited beds in hospitals, or there is not enough medicine for treatments. We have shown in Corollary 4.3 that backward bifurcations occur because of the insufficient capacity of treatment. We have also shown that (3) has bi-stable endemic equilibrium because of the limited resources. This means that driving the basic reproduction number below 1 is not enough to eradicate the disease. The level of initial infectious invasion must be lowered to a threshold so that the disease dies out or approaches a lower endemic steady state for a range of parameter 


\section{References}

[1] Wang W., 2006. Backward Bifurcation of an Epidemic Model with Treatment, J. Math. Bio. Sci., 201:58-71.

[2] Capasso V. and G. Serio., 1978. A Generalization of the Kermack-Mckendrick Deterministic Epidemic Model. Mat,. Bio. Sci., 42:43-61.

[3] Derrick W.R. and P. Van Der Driessche ., 1993. A Disease Transmission Model in a Non- constant Population, J. Math. Biol., 31:495-512.

[4] Esteva L. and M. A. Matias., 2001. Model for Vector Transmitted Diseases with Saturation Incidence, Journal of Biological System, 9(4):235-245.

[5] Gajendra Ujjainkar, V. K. Gupta, B. Singh, R. Khandelud., 2012. An Epidemic Model with Modified Non-monotonic Incidence Rate under Treatment, 6:1159-1171.

[6] Hethcote H.W. and P. Van Den Driessch., 1991. Some Epidemiological models with Non- linear Incidence, J. Math. Boil., 29:271287 .

[7] Jasmine D. and E.C.Henry Amirtharaj., 2013. Modeling and Simulation of Modified SIR Epidemic Model with Immigration and Non-monotonic Incidence Rate under Treatment, Indian Journal of Applied Research, 3(7):453-454.

[8] Jasmine D. and E. C. Henry Amirtharaj., 2013. Global Analysis of SIR Epidemic Model with Immigration and Non-monotonic Incidence Rate, International Journal of Applied Mathematics and Statistical Sciences, 2(5):83-92.

[9] Jasmine D. and Henry Amirtharaj E.C., 2013. Modeling and Simulation of Modified SIR Epidemic Model with Immigration and Non-monotonic Incidence under Treatment, Indian Journal of Applied Research, 3(7):43-44.

[10] Jasmine D. and E. C. Henry Amirtharaj., 2013. Global Analysis of SIR Epidemic Model with Immigration and Non-monotonic Incidence Rate under Treatment, International Journal of Scientific Research, 9(2):09-18.

[11] Kar T.K. and Ashim Batabyal., 2010. Modeling and Analysis of an Epidemic Model with Non-monotonic Incidence Rate under Treatment, Journal of Mathematical Research, 2(1):103-115.

[11] Liu W.M. and H. W. Hethcote and S. Levin., 1987. A Dynamical Behavior of Epidemiological Models with Non-linear Incidence Rates, J. Math. Biol., 25:359-380.

[12] Liu W.M., S. A. Levin and Y. Iwasa., 1986. Influence of Non-linear Incidence Rates upon the Behavior of SIRS Epidemiological Models, J. Math. Biol., : 187-204.

[13] Mena Lorca J. and H. W. Hethcote., 1992. Dynamic Models of Infection Diseases as Regulators of Population Size, J. Math. Bio., 30:693-716.

[14] Ruan S. and W. Wang., 2003. Dynamical Behavior of an Epidemic Model with Non-linear Incidence Rate, J. Differential Equations, 188:135-163.

[15] Xiao D. and S. Ruan., 2007. Global Analysis of an Epidemic Model with Non-monotone Incidence Rate, Math. Bio.. Sci. 208:419429. 